\title{
Antibiotic Prophylaxis at the Time of Dental Implant Placement: A Cost/Effectiveness Analysis
}

\author{
Khrystyna Zhurakivska ( $\square$ khrystyna.zhurakivska@gmail.com ) \\ Universita degli Studi di Foggia https://orcid.org/0000-0002-2153-0778 \\ Lucio Lo Russo \\ University of Foggia: Universita degli Studi di Foggia \\ Lorenzo Lo Muzio \\ University of Foggia: Universita degli Studi di Foggia \\ Vito Carlo Alberto Caponio \\ University of Foggia: Universita degli Studi di Foggia \\ Luigi Laino \\ University of Campania Luigi Vanvitelli: Universita degli Studi della Campania Luigi Vanvitelli \\ Claudia Arena \\ University of Foggia: Universita degli Studi di Foggia \\ Giuseppe Troiano \\ University of Foggia: Universita degli Studi di Foggia https://orcid.org/0000-0001-5647-4414
}

\section{Research Article}

Keywords: cost-effectiveness, antibiotics, antibiotic prophylaxis, implants, dental implant

Posted Date: February 18th, 2022

DOI: https://doi.org/10.21203/rs.3.rs-880083/v1

License: (c) (i) This work is licensed under a Creative Commons Attribution 4.0 International License. Read Full License 


\section{Abstract \\ Background}

antibiotic prophylaxis when performing implant placement seems to improve implant short term survival. Nevertheless, use of antibiotics carries risks of adverse effects and increasing antibiotic resistance. The aim of present study is to compare in terms of costs and effectiveness the use of antibiotics in dental implant procedures.

\section{Methods}

a decision-tree model with a short time horizon of 1 year was developed using TreeAge Pro Healthcare software. Two strategies were compared: Antibiotics and No Antibiotics in implant placement procedures. The costs were calculated considering direct costs for implant placement, antibiotic costs, and costs for implant replacement in case of failure. Effectiveness was defined in terms of General Oral Health Assessment Index, outcomes were evaluated as Incremental Cost Effectiveness Ratio. One-way sensitivity analysis and Probabilistic Sensitivity Analysis were performed for the most influential variables to test the parameters uncertainty. Patients and societal perspectives were considered.

\section{Results}

antibiotic prophylaxis resulted as the dominating strategy, compared with the no use of antibiotics. The cost of antibiotics, cost of implant replacement in case of failure and the probability of adverse effects significantly influence the result.

\section{Conclusions}

from single patient's perspective, antibiotic strategy can be considered cost-effective, even if the cost of antibiotic therapy increases. We can conclude that in clinical practice the administration of antibiotics in association with implant placement is recommended, as it increases the success rate and makes the treatment more effective, but attention should be placed when societal impact is considered in terms of antibiotic resistance that may impact the public health.

\section{Background}

Implant placement is a surgical procedure, aiming to rehabilitate edentulous areas of the jaws [1]. The surgical methodology has been highly standardized, reaching an optimum level of predictability. However, in some cases, the process of osseointegration can be undermined by several factors, among which intraoperative or postoperative infections [2, 3]. Antibiotic prophylaxis at the time implant placement has been advocated by several authors, proposing various molecules and regimens of administration $[4,5]$. However, the real effect of antibiotic use on implant survival and incidence of infectious complications is still unclear. A recent review and meta-analysis synthetized scientific findings regarding the influence of antibiotic prophylaxis on early implant failure rate, showing a statistically significant improvement in patients treated by means of antibiotic therapy [6]. On the other hand, the antibiotics, cannot be indiscriminately prescribed, due to their adverse effects and, above all, the increasing worldwide problem of antibiotic resistance [7]. For these reasons, only procedures that really benefit from antibiotic treatment should be associated to their use. This dilemma gives rise to necessity of a careful comparison of costs and benefits about the use of these class of drugs.

Cost-effectiveness analysis (CEA) is an analytic approach aiming at comparison of two or more alternative courses in terms of resource consumption and health outcomes. The result of such analysis is fundamental for an evidence-based decision making and an optimal allocation of health resources [8]. Costs, as well as, outcomes to be considered can vary depending on the perspective of analysis. In dentistry, one of the following perspectives is usually used when conducting a CEA: society, third-party payer (insurance company), dental practitioner or patient population [9].

The purpose of present study was to investigate whether the incremental cost of antibiotic prophylaxis during dental implants placement was justified by a consistent improvement in success rate of implant survival. Considering that Italian healthcare system does not cover dental procedures, and the dentistry is paid by the patient to a private clinician, the analysis has been performed from a single-patient's perspective. Furthermore, a consideration of societal perspective was made and discussed.

To our best knowledge, this is the first study investigating the economic aspect of antibiotic administration during dental implant placement.

\section{Methods}

The manuscript has been structured following standard recommendations for reporting of health economic evaluations (CHEERS) [10]. The two evaluated strategies referred to implant placement with or without contextual antibiotic intake, both patients and societal perspectives were 
analyzed by assessing Incremental cost-effectiveness ratios (ICERs).

\section{Population}

The model was set to reproduce a hypothetical situation in which a patient, in a good general health status, requires implant rehabilitation of a missing tooth. Personal characteristics such as age and sex were considered not influencing the outcome, based on results of available studies [4, 11-14]. Patients with comorbidities requiring antibiotic prophylaxis (like immunosuppressed conditions, infective endocarditis, metabolic disorders, and patients with prosthetic joints) were not considered in this analysis, since the benefit obtained from antibiotic assumption is supposed going beyond the implant survival rate.

The intervention is related to the replacement of a posterior tooth (without considering aesthetic value of the rehabilitation) in native bone, without any prior or contextual use of synthetic biomaterial. The treatment was estimated to be performed in Italy by dentist in a private practice setting during 2021.

\section{Model structure}

A decision tree with a short time horizon of 1 year (12 months) was built using TreeAge Pro Healthcare 2021 software (Fig. 1). Such a time horizon was chosen since it was deemed long enough to capture relevant changes and early success of implant placement and integration.

\section{Model assumptions}

An assumption was made based on authors judgement that the choice of implant replacement, in case of first implant failure, is made within the same year. No consideration about antibiotics use was made for implant replacement procedure, since the literature is lacking on this specific topic and the available data do not specify if the success rate of replaced implants is associated with the use of antibiotics [15].

\section{Data sources for model inputs and variables}

\section{Probabilities}

Data regarding survival rates of implants were obtained from a recently published systematic review and meta-analysis investigating the role of antibiotics in early implant failure, in which a Risk ratio of 0.31 has been reported for procedures associated with antibiotic prophylaxis compared to implant placement without antibiotic assumption [6]. Studies included in such a meta-analysis showed cohorts of patients from different countries and multiple implant brands. No specific information is available about patients' demographic data, despite that they were in good health status.

The probability of implant replacement in case of failure was assumed to be of $85 \%$, with a survival rate of $89 \%$, according to Troiano et al. [15] in case of immediate implant replacement.

Regarding antibiotic prophylaxis, single course Amoxicillin (1000 mg every 12 hours for 5 days) or 2000mg of Amoxicillin prior surgery was considered in its direct costs and costs of adverse effects (AE). The most diffused AE were considered: allergy, cutaneous rushes, gastrointestinal mild to severe manifestations, anaphylaxis. The probability of these non-fatal adverse effects of single course was set at 0.000023 , and probability of fatal AE was set at 0.00 , according to Thornhill et al [16].

\section{Costs}

Costs of implant placement were obtained from database sourced by statista.com[17] reporting average costs of dental implant in private practice in Italy referred to 2018.

The cost of single course of Amoxicillin was estimated in $€ 10.20$, based on average dispensing costs.

The costs of AE management were estimated considering medication prescription for mild-moderate symptoms (antihistamine or antidiarrheal) and hospitalization costs for anaphylaxis and severe gastrointestinal AE. The direct cost of antihistamine/antidiarrheal preparations was assumed to be of $10.00 €$ per 2 days treatment. In case of severe AEs, hospitalization costs are assumed to be fully covered by the national health system (Italy). So, when healthcare payer's perspective was considered, Diagnosis-related group (DRG) codes were used to estimate costs of hospitalization. In particular, DRG 447 code was used for allergic reactions treatment, with the cost of $€ 1404.00$ stated by official Italian government based on the DRG document 2013 [18].

A separate consideration was made to estimate the cost of Antibiotic resistance. The hidden societal costs of antibiotic resistance were quantified to be equivalent to $\$ 13$ for each ambulatory antibiotic prescription in United States, referred to 2013 [19]. Another study estimated the cumulative cost of antimicrobial resistance to be $9,3 \$$ per single course of broad spectrum penicillin consumed in United states [20]. An average value of $11,15 \$$ was chosen for the analysis; amounts were converted to EUR to adjust for purchasing power parity at USD1 = EUR0.85. In the paper of

Page $3 / 12$ 
Shrestha [20] both the direct and indirect costs were considered in the calculation of Antibiotic resistance cost. Indirect costs included an estimation of productivity losses due to excess morbidity and premature mortality.

All costs were adjusted for inflation to 2021 (1,8\%) and were all annual costs. Inflation rates were obtained from Eurostat database (https://ec.europa.eu/eurostat/).

\section{Effectiveness}

The effectiveness of each strategy was valued based on data available in literature. In particular, data from the study of Korenori et al. [21], resulting from questionnaire survey for the General Oral Health Assessment Index (GOHAl) were used. GOHAI is a specific oral health assessment index that express the QOL related to oral cavity. GOHAl comprises three physical areas: eating, swallowing and pronunciation and two psychosocial aspects, consisting of aesthetic appreciation and sociability. Furthermore, pain and discomfort are evaluated through the investigation of medicines use and hypersensitivity evaluation. The total score is expressed in the range of $12-60$, but for our purposes the GOHAI value was available in the continuous values between 0 and 1 based on beta distribution ( 0 : no satisfaction; 1: maximum satisfaction). The established utilities were 0.88 for implant rehabilitation and 0.71 for implant loss, like emerged from Korenori et al. study [21]. Considering a time horizon of one year, the above mentioned utilities were used for qualitative effectiveness evaluation and no quantitative calculation of Qualityadjusted life years was made.

\section{Sensitivity analysis}

An influence analysis was performed to rank variables in order of their influence on the magnitude of the model output. Then, one-way sensitivity analysis was performed for the most influential variables to test parameters uncertainity. The tested ranges were decided based on expert's opinion and are listed on Table 1. In order to test the impact of overall uncertainty For sensitivity analysis, WTP threshold was set at $3000 €$, based on data reported in studies investigating the patients' preferences for implant rehabilitation of single-tooth gap in Italy [22]. Variables were defined for each parameter considered in the analysis. Input values assigned to the variables, their ranges and sources are summarized on Table 1.

\section{Results}

Antibiotic strategy resulted to be dominating from both perspectives, since less expensive $(1023,64 €$ for patient's and $1037,61 €$ for societal perspectives Vs 1042,56€ for no antibiotic approach) and slightly more effective (0,87949 vs 0,87821 GOHAI), when compared to No Antibiotic approach with Incremental Cost-Effectiveness Ratio of 14692,64 for patient's and 3841,18 for societal perspectives. The costs, effectiveness, ICERs and NMBs resulted from examination of both perspectives are reported on Table 2 . The only difference between the patients and societal perspectives is represented by the cost of Antibiotic resistance that, in case of Italy falls on healthcare provider that is covered by societal health system.

Sensitivity analysis performed from societal perspective showed relevance of three variables: cost of antibiotics, cost of implant replacement and probability of adverse effects for dominance persistence. In particular, cost of antibiotics, probability of adverse effects and cost of antibiotic resistance are inversely proportional with Antibiotic strategy dominance and at hypothetical thresholds of 18,59€ for the cost of Antibiotics, a probability of adverse effects greater than 0,01 , and a cost of antibiotic resistance greater than $22,36 €$ the two strategies become neutral for a WTP of $3000 €$.

The cost of implant replacement, conversely, is directly correlated with Antibiotic strategy dominance, since a great price of Implant replacement, encourage Antibiotic administration to avoid implant failure. In particular, for a cost of Implant replacement higher than $754,04 €$, the Net Monetary Benefit of Antibiotic strategy become positive.

Results of one-way sensitivity analysis for the most relevant variables are graphically represented in Figure 2 and threshold reports of sensible variables are shown on Table 3. Variables mostly influencing the relative cost-effectiveness of the strategy were: Costs of Antibiotics, Cost of Implant replacement, Probability of Adverse effects, Probability of survival without antibiotic prophylaxis and probability of Implant replacement. The ranking of variables influencing ICER is graphically represented in Tornado diagram (Fig. 3).

PSA performed with 1000 Monte Carlo simulations confirmed the dominance of Antibiotic strategy. An illustration of uncertainty based model calculation is presented in the cost/effective Scatterplot of Figure 4. Acceptability curves analysis revealed that the Antibiotic strategy remains dominant for each value of WTP ranging from 0 to $20000 €$.

\section{Discussion}

Antibiotic prophylaxis is recommended in dental everyday practice in some specific clinical conditions, among which: treatment of acute odontogenic and non-odontogenic infections and prevention of local and focal infections in susceptible patients during invasive procedures [23]. However, in clinical setting, there is often an abuse of antibiotics prescription, neglecting the risk of adverse effects and antibiotic resistance that represent an increasing public health problem [24]. Among dental interventions for which antibiotic prophylaxis is often suggested, there is implant surgery. The prescription of antibiotics is justified by the fact that implant insertion is an invasive surgical procedure that is performed in

Page $4 / 12$ 
an infected environment (oral cavity) and involves the insertion of a foreign body for which an integration with the surrounding tissues is expected. A recent meta-analysis confirmed a slightly greater short-term success of implant rehabilitation in patients undergoing antibiotic prophylaxis, compared to patients who did not undertake antibiotics during implant placement [6]. However, this conclusion refers only to the reduction of implant failures and does not take into account other aspects associated with antibiotics usage. A recent article estimated that the total societal cost of antibiotic resistance attributable to each ambulatory antibiotic prescription in US is about \$13 [19]. Another study estimated the economic costs of antimicrobial resistance in Thailand and United States, based on type of responsible pathogen and antibiotic class driving resistance [20]. The presented data are assumed to be valid for both the low/middle and high-income countries. The costs associated with antimicrobial resistance were considered from several points of view: patient, healthcare and societal perspectives, being responsible of increase of morbidity and mortality, earnings loss, higher toxicity of 2 nd line drugs, longer hospital admissions, development of alternative drugs etc. For use in our analysis we considered the resistance costs estimated for broad spectrum penicillin in US. Nevertheless, the complexity of such an estimation due to variation in populations, drugs, healthcare system and societal organization should be considered and an appropriate sensitivity analysis should be always undertaken to verify the robustness of the model. The purpose of this analysis was to integrate biological and economic costs and benefits deriving from the use of antibiotics in patients undergoing implant surgery and compare them with no use of antibiotics. The time horizon for decision analysis was set at 1 year, since it was deemed long enough to capture relevant changes and outcomes of implant therapy. Sources of parameters considered in this study were various, including: meta-analysis, original studies, electronic databases. Being aware of the variable robustness of each source, uncertain data and assumptions were then addressed in sensitivity analysis. Since dental procedures are mainly private in Italy and costs are supported directly by patients, patient's perspective has been examined. Nevertheless, since some costs deriving from the use of antibiotics (severe adverse effects management, antibiotic resistance consequences) may involve the state health system, a societal perspective has also being evaluated. Both perspectives revealed a net dominance of antibiotic prophylaxis, since it resulted to be both cheaper and more effective (ICER equal to 3292,44 and 14672,1€ per point of gained GOHAI for patient's and societal perspectives respectively). Productivity loss was not considered in costs evaluation, since implant placement is an outpatient intervention, which usually takes place in a few hours and does not require long hospital stays. Therefore, it shouldn't affect patients' work or personal life very much. Furthermore, if it had been considered, it would certainly have increased the convenience of the antibiotic strategy even more, as it would have reduced the possibility of having to undergo implant replacement in case of implant failure. The most difficult parameter to determine was the QOL related to implant rehabilitation, since available data regarding oral health related QOL are variable [25]. GOHAI was used for the indicator of effectiveness as an oral health QOL value, as suggested by some studies $[21,26]$. Values assumed for assessment of efficacy $(0.88$ and $0.71 \mathrm{for}$ implant rehabilitation and lost implant respectively) refer to a specific situation of single molar implant rehabilitation. In case of anterior implant placement, the utility measure can vary significantly, since aesthetic factors should be considered. However, even in this case, the importance of implant success would increase even more, and therefore the effectiveness of antibiotic therapy. GOHAl is a widely used index for oral healthrelated quality of life (OHRQOL) evaluation. It was chosen for utility measurement because deemed to be sensitive enough to capture a small change in health status, like that represented by single-tooth implant-supported rehabilitation. We are aware the use of such score is not ideal to weight for the construction of Quality-adjusted life-years. Nevertheless, since the time horizon is one year, Utility values can be only used for OHRQOL measure.

Sensitivity analysis revealed as the cost of antibiotics, the cost of implant replacement in case of failure and probability of adverse effects are the most relevant factors in determining dominant strategy. In particular, if the cost of antibiotics (including the estimated cost of antibiotic resistance) exceeded 20,15 euros, the antibiotic prophylaxis would loss its convenience in terms of ICER. This threshold value is much higher, when compared with the dispensing costs of antibiotics (about €10) for the patient, nevertheless it could be achieved if the social cost is considered. The same inversion of convenience would happen if the probability of adverse effects was significantly higher (>0.01) or the cost of implant replacement was significantly lower $(<€ 754,04)$.

Regarding the willingness to pay, the threshold of $3000 €$ was estimated to be valid for both the patient's and societal perspectives. From societal perspective, it is difficult to estimate what is the societal benefit from single-tooth replacement and how much of additional taxes patients are available to pay for supporting the public treatment and all the associated costs. Nevertheless, in our case the antibiotic strategy resulted to be both less expensive and more effective even if the WTP was $0 €$.

The main limitations of the study are related to a close connection with the specific context represented by Italian healthcare organization. This aspect limits the generalizability of both results and conclusions. Nevertheless, the consideration of a wider societal perspective allowed us to expand the possible applicability of the model. The second limitation of the study regards some sources of the values used for defining the variables. In particular, the effectiveness estimation and some probabilities were obtained from original papers. Meanwhile, some other measures derived from meta-analysis, representing more reliable sources. This issue was determined by the fact that few data are available for such a specific condition like single-tooth replacement by the implant. Furthermore, the utility evaluation was obtained from a paper evaluating Japanese population OHRQOL. So, this setting may be slightly different for Italian population, even if no big differences in terms of health status exists between Japan and Italy [27]. To address this issue, wide ranges of values were used in sensitivity analysis in order to test the uncertainty of the values. 


\section{Conclusions}

The use of antibiotics during implant placement resulted to be a cost-effective strategy, compared with no use of antibiotics. However, the dominance of the strategy involving the use of antibiotics is sensitive to the cost of antibiotics and the cost of a possible implant replacement. From single patient's perspective, antibiotic strategy can be considered cost-effective, even if the cost of antibiotic therapy increases, but attention should be placed when societal impact is considered, since the costs of management of antibiotic resistances supported by healthcare system may significantly undermine the certainty of the choice.

\section{Abbreviations}

CEA: Cost-effectiveness analysis;

ICERs: Incremental cost-effectiveness ratios;

AE: adverse effects;

Diagnosis-related group (DRG);

GOHAl: General Oral Health Assessment Index;

ISTAT: Italian National Institute of Statistics;

QOL: quality-of-life;

WTP: Willingness to pay.

\section{Declarations}

Ethics approval and consent to participate: "This article does not contain any studies with human participants or animals performed by any of the authors".

Consent for publication: Not applicable.

Availability of data and material: "All the data are available from the corresponding author after reasonable request".

Competing interests: None.

Funding: None.

Authors' contributions: All authors have read and approved the manuscript.

K.Z. and G.T designed and wrote the study; VCAC and LL performed data analysis, CA performed data extraction; LLR and LLM critically revised the manuscript.

Acknowledgements: None.

\section{References}

1. Brånemark PI. Osseointegration and its experimental background. J Prosthet Dent. 1983;50(3):399-410.

2. Sakka S, Baroudi K, Nassani MZ. Factors associated with early and late failure of dental implants. J Investig Clin Dent. 2012;3(4):258-61.

3. Wismeijer D, Chen ST: Proceedings of the 6th ITI Consensus Conference. Clin Oral Implants Res 2018, 29 Suppl 16:5-7.

4. Nolan R, Kemmoona M, Polyzois I, Claffey N. The influence of prophylactic antibiotic administration on post-operative morbidity in dental implant surgery. A prospective double blind randomized controlled clinical trial. Clin Oral Implants Res. 2014;25(2):252-9.

5. El-Kholey KE. Efficacy of two antibiotic regimens in the reduction of early dental implant failure: a pilot study. Int J Oral Maxillofac Surg. 2014;43(4):487-90.

6. Canullo L, Troiano G, Sbricoli L, Guazzo R, Laino L, Caiazzo A, Pesce P. The Use of Antibiotics in Implant Therapy: A Systematic Review and Meta-Analysis with Trial Sequential Analysis on Early Implant Failure. Int J Oral Maxillofac Implants. 2020;35(3):485-94.

7. Kern WV. Organization of antibiotic stewardship in Europe: the way to go. Wien Med Wochenschr. 2021;171(Suppl 1):4-8.

8. Sanders GD, Neumann PJ, Basu A, Brock DW, Feeny D, Krahn M, Kuntz KM, Meltzer DO, Owens DK, Prosser LA, et al. Recommendations for Conduct, Methodological Practices, and Reporting of Cost-effectiveness Analyses: Second Panel on Cost-Effectiveness in Health and 
Medicine. JAMA. 2016;316(10):1093-103.

9. Sendi PP, Palmer AJ, Marinello C. Some principles of cost-effectiveness analysis in dentistry. Acta Med Dent Helv. 1999;4(4):63-7.

10. Husereau D, Drummond M, Petrou S, Carswell C, Moher D, Greenberg D, Augustovski F, Briggs AH, Mauskopf J, Loder E. Consolidated health economic evaluation reporting standards (CHEERS)-explanation and elaboration: a report of the ISPOR health economic evaluation publication guidelines good reporting practices task force. Value in health. 2013;16(2):231-50.

11. Caiazzo A, Casavecchia P, Barone A, Brugnami F. A pilot study to determine the effectiveness of different amoxicillin regimens in implant surgery. J Oral Implantology. 2011;37(6):691-6.

12. Esposito M, Cannizzaro G, Bozzoli P, Checchi L, Ferri V, Landriani S, Leone M, Todisco M, Torchio C, Testori T. Effectiveness of prophylactic antibiotics at placement of dental implants: a pragmatic multicentre placebo-controlled randomised clinical trial. Eur $\mathrm{J}$ Oral Implantol. 2010;3(2):135-43.

13. Esposito M, Cannizzaro G, Bozzoli P, Consolo U, Felice P, Ferri V, Landriani S, Leone M, Magliano A, Pellitteri G. Efficacy of prophylactic antibiotics for dental implants: a multicentre placebo-controlled randomised clinical trial. European journal of oral implantology 2008, 1(1).

14. Tan WC, Ong M, Han J, Mattheos N, Pjetursson BE, Tsai AYM, Sanz I, Wong MC, Lang NP, Group IAS. Effect of systemic antibiotics on clinical and patient-reported outcomes of implant therapy-a multicenter randomized controlled clinical trial. Clin Oral Implants Res. 2014;25(2):18593.

15. Troiano G, Fau - Luongo R, Luongo R, Fau - Romano DC, Romano D, Fau - Galli M, Galli M, Fau - Ravidà A, Ravidà A, Fau - Wang H-L, Wang H, Fau - Laino L, Laino L. Comparison of immediate versus delayed implant placement in a failed implant site: A retrospective analysis of early implant survival FAU - Troiano, Giuseppe. (2631-6420 (Print)).

16. Thornhill MH, Dayer MJ, Prendergast B, Baddour LM, Jones S, Lockhart PB. Incidence and nature of adverse reactions to antibiotics used as endocarditis prophylaxis. J Antimicrob Chemother. 2015;70(8):2382-8.

17. Average price of a dental implant in Italy 2018. https://www.statista.com/statistics/971051/average-price-for-a-dental-implant-in-italy/.

18. Diagnosis related groups [https://www.anticorruzione.it/portal/rest/jcr/repository/collaboration/Digital\%20Assets/Pdf/CIG700397355C/All.\%201\%20DM\%2018.10.12GU\%2028.1.13].

19. Michaelidis Cl, Fine MJ, Lin CJ, Linder JA, Nowalk MP, Shields RK, Zimmerman RK, Smith KJ. The hidden societal cost of antibiotic resistance per antibiotic prescribed in the United States: an exploratory analysis. BMC Infect Dis. 2016;16(1):1-8.

20. Shrestha P, Cooper BS, Coast J, Oppong R, Do Thi Thuy N, Phodha T, Celhay O, Guerin PJ, Wertheim H, Lubell Y. Enumerating the economic cost of antimicrobial resistance per antibiotic consumed to inform the evaluation of interventions affecting their use. Antimicrob Resist Infect Control. 2018;7:98.

21. Korenori A, Koji K, Yuki T, Murata T, Sachiko TM, Shunsuke B. Cost-effectiveness of molar single-implant versus fixed dental prosthesis. BMC Oral Health. 2018;18(1):141.

22. Augusti D, Augusti G, Re D. Prosthetic restoration in the single-tooth gap: patient preferences and analysis of the WTP index. Clin Oral Implants Res. 2014;25(11):1257-64.

23. Ahmadi H, Ebrahimi A, Ahmadi F. Antibiotic Therapy in Dentistry. Int J Dent. 2021;2021:6667624.

24. Sukumar S, Martin F, Hughes T, Adler CJ. Think before you prescribe: How dentistry contributes to antibiotic resistance. Aust Dent J. 2020;65(1):21-9.

25. Beecher T, James P, Browne J, Di Blasi Z, Harding M, Whelton H. Dental patient reported outcome and oral health-related quality of life measures: protocol for a systematic evidence map of reviews. BDJ open. 2021;7(1):1-6.

26. Teranishi Y, Arai K, Baba S. Cost-Utility Analysis of Molar Single Implant Versus Fixed Dental Prosthesis. Int J Prosthodont. 2019;32(1):75-81.

27. James SL, Abate D, Abate KH, Abay SM, Abbafati C, Abbasi N, Abbastabar H, Abd-Allah F, Abdela J, Abdelalim A, et al. Global, regional, and national incidence, prevalence, and years lived with disability for 354 diseases and injuries for 195 countries and territories, 1990-2017: a systematic analysis for the Global Burden of Disease Study 2017. The Lancet. 2018;392(10159):1789-858.

\section{Tables}

Table 1: Description of variables, values, sources and tested ranges. 


\begin{tabular}{|c|c|c|c|c|c|c|}
\hline Variables & Description & ${ }_{\text {Reot }}^{\text {Definition }}$ & Source & $\begin{array}{l}\text { Costs adjusted } \\
\text { for inflation }\end{array}$ & Low & $\underline{\text { High }}$ \\
\hline CAE & $\begin{array}{l}\text { cost Adverse effects of } \\
\text { antibiotics }\end{array}$ & 705 & $\begin{array}{l}\text { DRG, Health ministery of } \\
\text { Italy[18] (2013) }\end{array}$ & 708.6 & 10 & 1800 \\
\hline cAntibiotics & Costs of antibiotics & 10.20 & $\begin{array}{l}\text { Assumed based on } \\
\text { dispensing costs } 2021 \text {. }\end{array}$ & 10.20 & 5 & 100 \\
\hline cAntibioticResistance & Cost of antibiotic resistance & 9.47 & $\begin{array}{l}\text { Michaelidis et al.; Shrestha } \\
\text { et al. }[19,20]\end{array}$ & 13.97 & 2.9 & 32.16 \\
\hline clmplantPlacement & Cost of Implant Placement & 1002 & $\begin{array}{l}\text { Statista.com[17] } \\
(2018)\end{array}$ & 1002 & 500 & 1500 \\
\hline clmplantReplacement & Cost of Implant Replacement & 1102 & $\begin{array}{l}\text { Assumed based on Cost of } \\
\text { Implant Placement }\end{array}$ & 1102 & 0 & 1600 \\
\hline effimplant & $\begin{array}{l}\text { Effectiveness Implant } \\
\text { Placement }\end{array}$ & 0.88 & $\begin{array}{l}\text { Korenori et al.[21] } \\
\text { (2018) }\end{array}$ & & 0,5 & 0,9 \\
\hline effNolmplant & Effectiveness Lost implant & 0.71 & $\begin{array}{l}\text { Korenori et al.[21] } \\
\text { (2018) }\end{array}$ & & 0,3 & 0,8 \\
\hline pAE & $\begin{array}{l}\text { probability Adverse effects } \\
\text { antibiotics }\end{array}$ & 0.000023 & $\begin{array}{l}\text { Thornhill et al.[16] } \\
\text { (2015) }\end{array}$ & & 0,00001 & 0,05 \\
\hline pantsurv & $\begin{array}{l}\text { Probability of survival with } \\
\text { antibiotics }\end{array}$ & 0.9878 & $\begin{array}{l}\text { Canullo et al.[6] } \\
(2020)\end{array}$ & & 0,6 & 0,9878 \\
\hline pnoantsurv & $\begin{array}{l}\text { Probability of survival } \\
\text { without antibiotics }\end{array}$ & 0.9567 & $\begin{array}{l}\text { Canullo et al.[6] } \\
(2020)\end{array}$ & & 0,6 & 0,9567 \\
\hline preplac & $\begin{array}{l}\text { Probability of implant } \\
\text { replacement }\end{array}$ & 0.85 & Assumed & & 0,3 & 0,9 \\
\hline psurvsecond & $\begin{array}{l}\text { Probability of survival after } \\
\text { secondary placement }\end{array}$ & 0.89 & $\begin{array}{l}\text { Troiano et al.[15] } \\
\text { (2021) }\end{array}$ & & 0,6 & 0,89 \\
\hline
\end{tabular}

*The cost of antibiotic resistance was considered only for societal perspective.

Table 2: Incremental costs and effectiveness for each strategy

\begin{tabular}{|c|c|c|c|c|c|c|c|}
\hline Strategy & Cost & Incremental Cost & Effectiveness & Incremental E & ICER & NMB & \\
\hline \multicolumn{8}{|c|}{ Societal perspective } \\
\hline \multicolumn{7}{|c|}{ All referencing common baseline } & \multirow[b]{2}{*}{ undominated } \\
\hline Antibiotics & 1037,61 & & 0,879495 & & & 1600,87 & \\
\hline No Antibiotics & 1042,56 & 4,95 & 0,878208 & $-0,00129$ & $-3841,18$ & 1592,06 & abs. dominated \\
\hline \multicolumn{8}{|c|}{ Patient's perspective } \\
\hline \multicolumn{8}{|c|}{ All referencing common baseline } \\
\hline Antibiotics & 1023,64 & & 0,879495 & & & 1614,84 & undominated \\
\hline No Antibiotics & 1042,56 & 18,92 & 0,878208 & $-0,00129$ & $-14692,64$ & 1592,06 & abs. dominated \\
\hline
\end{tabular}

C: costs; E: effectiveness; abs: absolutely; NMB: Net Monetary Benefit; ICER: Incremental Cost-Effectiveness Ratio

Table 3: Threshold values of sensible variables. cAntibiotics: cost of antibiotics; clmplant_replacement: cost of implant replacement; pAE: probability of adverse events. 


\begin{tabular}{|llll|}
\hline Variable & Value & Tested range (min-max) & Threshold \\
\hline cAntibiotics & 24.85 & $5-100$ & 20,15 \\
\hline cImplant_replacement & 1101 & $0-1600$ & 770,53 \\
\hline pAE & 0.000023 & $0,00001-0,05$ & 0,01 \\
\hline
\end{tabular}

\section{Figures}

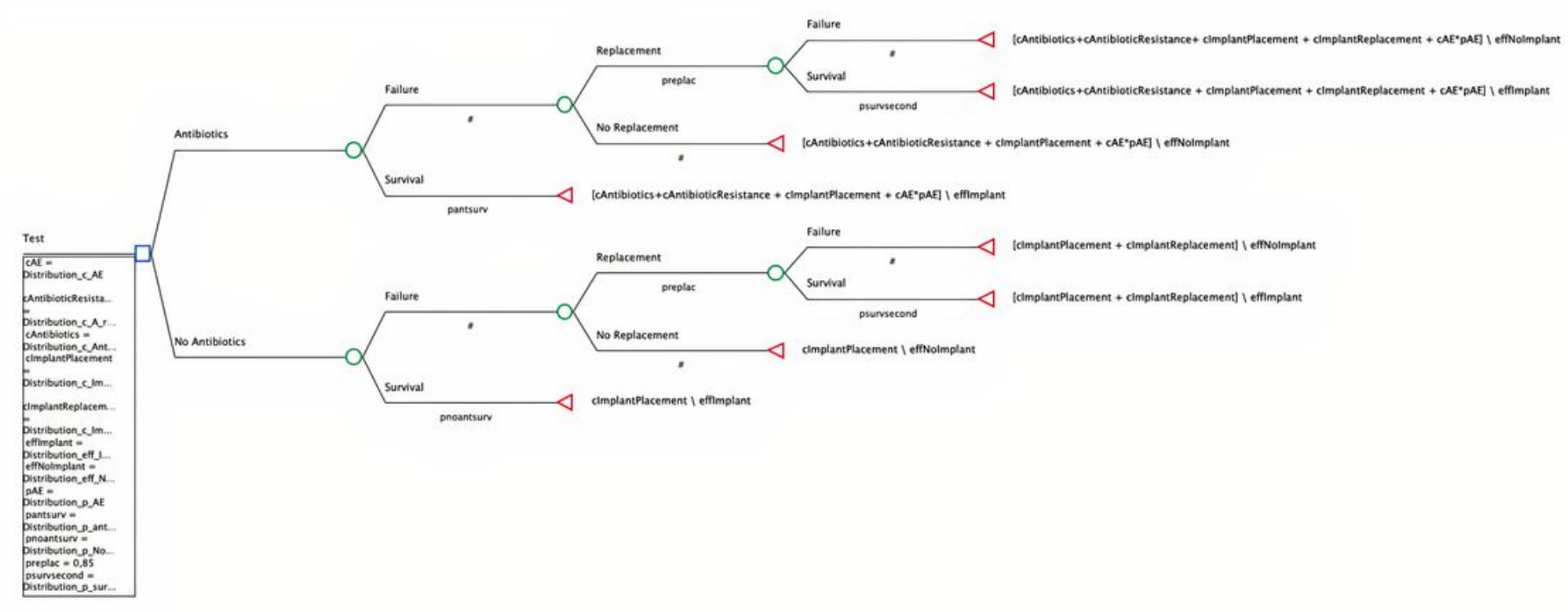

\section{Figure 1}

Model structure. The initial blue square box represents the decision node, from which two alternatives: Intervention (Antibiotics) and No intervention (No Antibiotics) branches depart. For each alternative two outcomes are possible: Failure or Survival, introduced by a chance node (green) and characterized by a certain probability. In case of Failure, another chance node has been placed introducing a potential Replacement of the implant with relative probabilities of Survival or Failure. Red triangles represent terminal nodes, where summary evaluations of payoffs, multiplied by probabilities, associated with each strategy of treatment are calculated. 

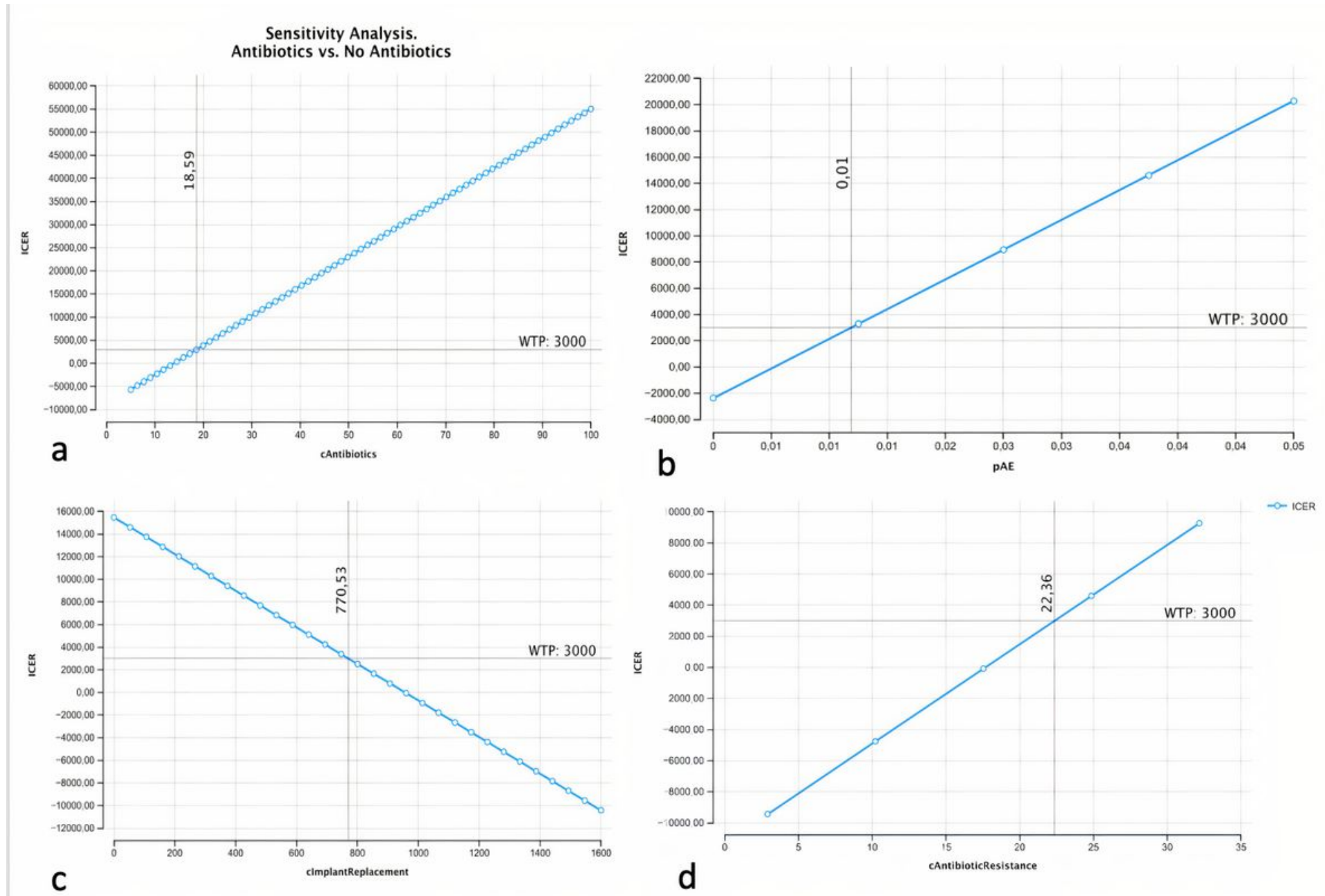

\section{Figure 2}

One-way sensitivity analysis of the most relevant variables. a) cost of antibiotics (cAntibiotics); b) probability of adverse effects (pAE); c) cost of implant replacement (clmplantReplacement); d) cost of Antibiotic resistance (cAntibioticResistance). 


\section{Tornado Diagram - ICER Antibiotics vs. No Antibiotics}

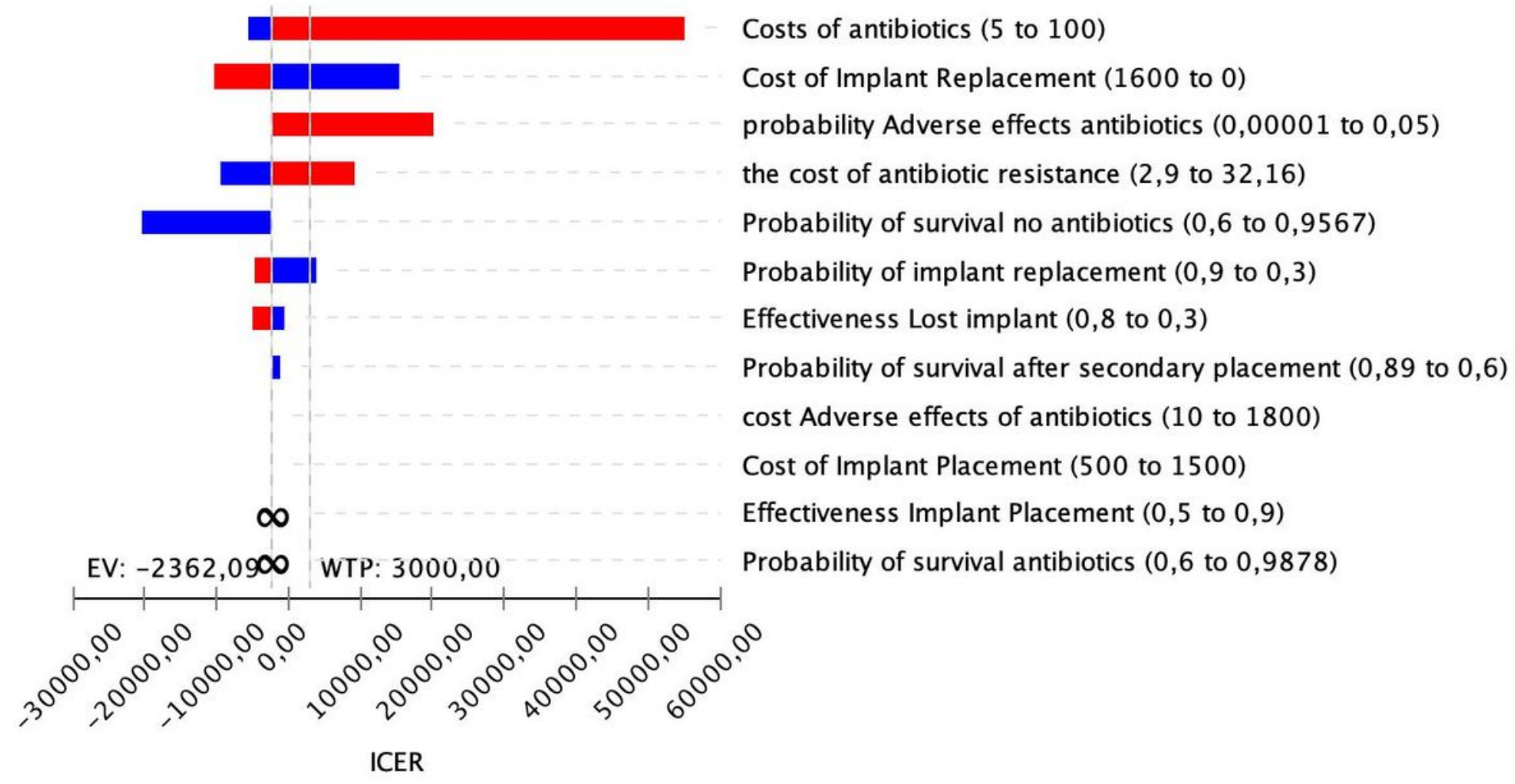

Figure 3

Tornado diagram. Tested variables are ranked in order of their overall influence on the magnitude of the model output. 


\section{Cost-Effectiveness Scatterplot}

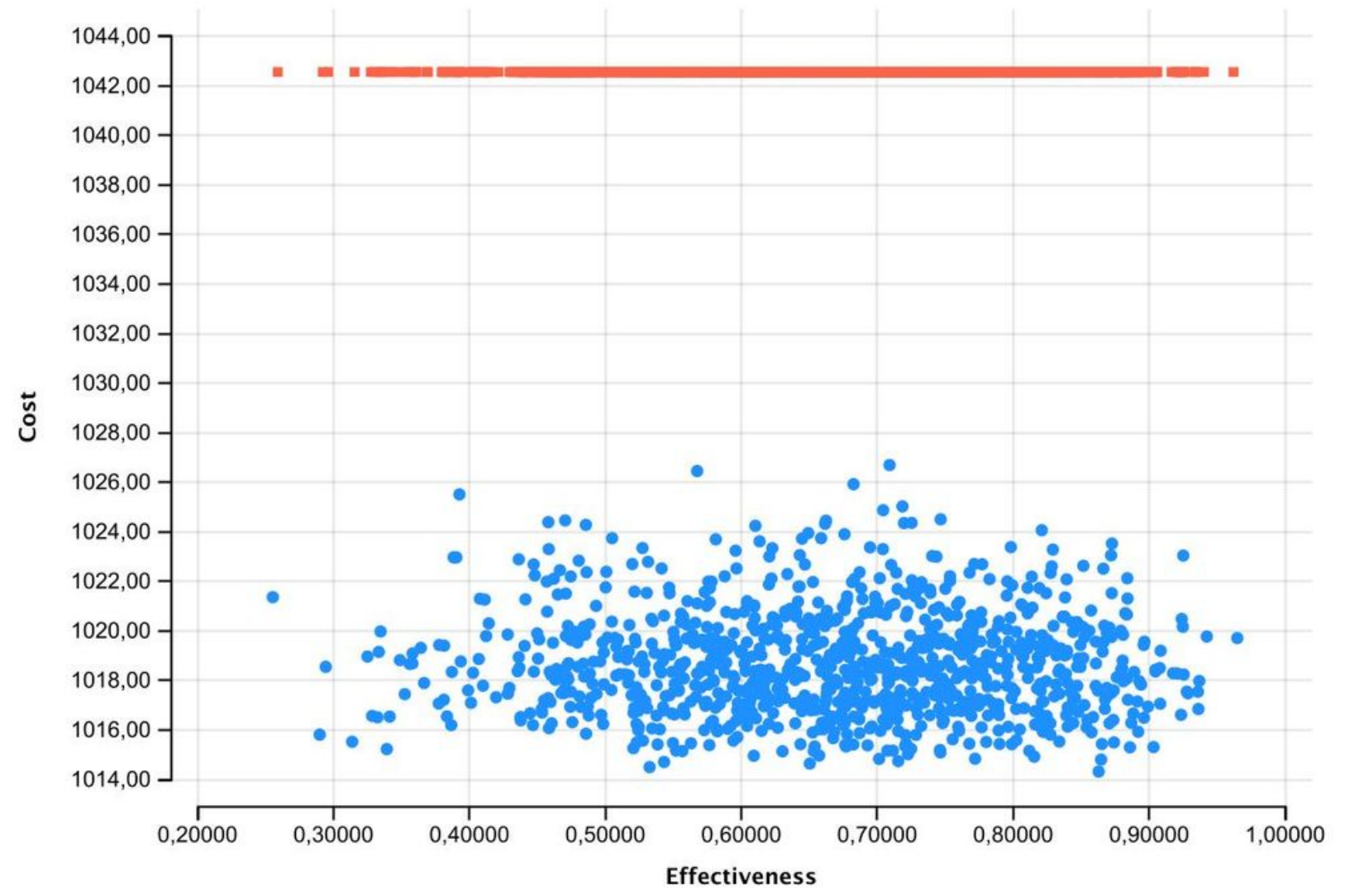

- Antibiotics

= No Antibiotics

\section{Figure 4}

CE scatterplot illustrating the distribution of calculations based on uncertainty of parameters.

\section{Supplementary Files}

This is a list of supplementary files associated with this preprint. Click to download.

- RevisedCHEERSChecklist.pdf 